\title{
Parallel Synthesis of 2-Sulphanylated Bis-benzimidazoles on Soluble Polymer Support
}

\author{
Chia-Mao Chang, ${ }^{\dagger}$ Manohar V. Kulkarni, ${ }^{\dagger}$ Chih-Hau Chen, ${ }^{\dagger}$ Chi-Hsiang Wang, ${ }^{\dagger}$ and \\ Chung-Ming Sun*,†
}

Department of Applied Chemistry, National Chiao Tung University, Hsinchu 300-10, Taiwan, ROC, and Department of Applied Science, National Taitung University, Taitung 950, Taiwan, ROC

Received December 29, 2007

\begin{abstract}
A well-sustained multistep synthetic protocol has been designed for the PEG-functionalized aromatic acid amide to generate a molecular library of 2-alkylthio bis-benzimidazoles. An attempted synthesis of benzimidazole-2-thiol in dichloromethane has led to $S$-chloromethyl methyl sulfides, mimicking bacterial enzymatic systems. Regioselective S-alkylation was brought about under controlled conditions using a mild base at room temperature. The polymer-free compounds, 2-sulfanylated bisbenzimidazoles, were obtained in high yields and high purities. Chemical shift changes in proton and carbon NMR have been employed to monitor the progress of the reaction steps and to prove the site of S-alkylation, respectively.
\end{abstract}

\section{Introduction}

Nitrogen-containing heterocyclic derivatives possess immense pharmaceutical significance, and the development of novel strategy for their synthesis is an important field of organic chemistry. ${ }^{1}$ Combinatorial synthesis of heterocycles provides enormous structural diversity, which has greatly helped in the design and choice of lead structures in the drug discovery process. ${ }^{2}$ The two established techniques in this field are solid- ${ }^{3}$ and liquid-phase synthetic strategies, ${ }^{4}$ employing a polymer support. Polyethylene glycol (PEG) is usually employed as the soluble polymer support in liquidphase synthesis. The PEG-monomer conjugates obtained are easily soluble in organic solvents, and the reactions can be monitored by traditional analytical techniques. Our group has been exploring this method to generate molecular libraries of diazepinones, fused hydantoins, piperazinones, pyrimidinethiones, quinoxalinones, and tricyclic carbolines. $^{5-10}$

The benzimidazole ring, classed as a privileged structure, is present in clinically approved anthelmintics, antiulcers, antivirals, antifungals, and antihistamines. ${ }^{11}$ Recently, there were reports on benzimidazole derivatives exhibiting antitumor and antimicrobial properties and acting as thrombopoietin receptor agonists. ${ }^{12}$ It is quite obvious that a large number of benzimidazoles have been synthetic targets for combinatorial approaches with the hope of delivering structures with improved pharmacological activity and lesser side effects. ${ }^{13,14}$

In recent years, two newer aspects connected with their biological properties have emerged. The first one is their

* To whom correspondence should be addressed. E-mail: cmsun@ mail.nctu.edu.tw.

National Chiao Tung University.

$\ddagger$ National Taitung University. ability to bind with the DNA minor groove found in bisbenzimidazoles, and the second is enzyme activity observed in 2-mercaptobenzimidazoles and their corresponding sulfides (Figure 1). ${ }^{15}$ The sequence-selective ability of bis-benzimidazoles to bind to the DNA minor groove has been enhanced by the bispyridyl derivatives and has also been subjected to computational analysis (Figure 1A). ${ }^{16}$ The origin of this effect lies in the optimum distance between the two nitrogens located in the two rings, and it has been linked with their antitumor activities through topoisomerase-I inhibition (Figure 1B). ${ }^{17,18}$ The pyridyl methyl sulfinyl benzimidazoles are well-known as in vivo and in vitro inhibitors of gastric $\left(\mathrm{H}^{+}-\mathrm{K}^{+}\right)$-ATP by covalent modification of the thiol group. ${ }^{19}$ The cyclic thioureide structure is also recognized for its thyroid peroxide inhibiting activity, which makes such compounds potential candidates for treatment of hyperthyroidism. ${ }^{20}$ Hepatic monooxygenases metabolize benzimidazolin-2-thione to benzimidazole, which is of immense significance in the design of thiocarbanilides with reduced toxicity. ${ }^{21}$ The 2-mercapto-1-(4-pyridylethyl) benzimidazole (MPB) is known to inhibit rRNA synthesis, which is responsible for its antiviral activity (Figure 1C). ${ }^{22}$ This compound has also been reported to induce melanogenesis in murine melanoma cells; this is useful in the differentiation of solid tumors. ${ }^{23}$ Recently, the crystal structure of the iodine adduct formed with benzimidazolin-2-thione was investigated for its mechanism of antithyroid action. ${ }^{24}$

Earlier we reported a microwave-assisted liquid-phase synthesis of the $S$-alkyl benzimidazole moiety. ${ }^{14 a-d}$ The design concept for the presently synthesized molecular library originated from the recognition of the biological role of the $S$-alkyl benzimidazole moiety. The generation of a combined bis-benzimidazole skeleton that resembles druglike molecules has a substantial intellectual appeal. Therefore, the biological 


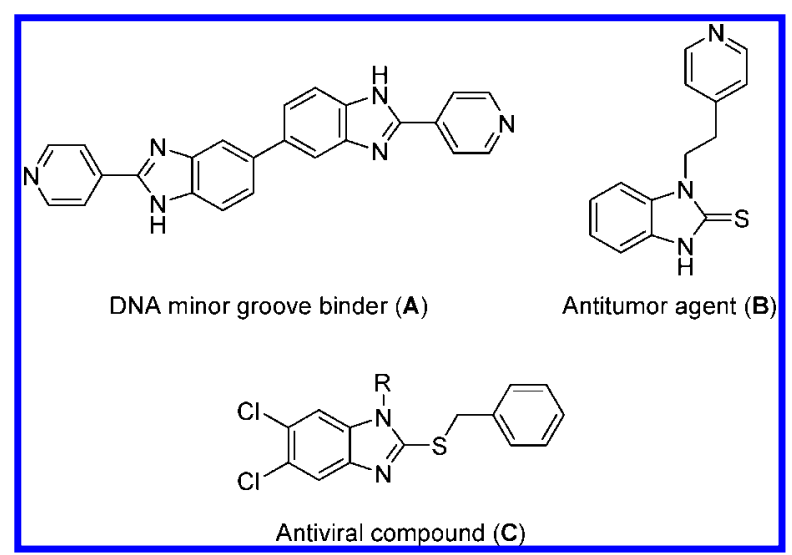

Figure 1. Biologically active benzimidazoles and sulfides.

potential of these two pharmacophores have been assembled into a single molecular library with three points of structural diversity.

\section{Results and Discussions}

The steps involved in the synthesis of bisbenzimidazolin2-thiones are outlined in Scheme 1. PEG (MW $\approx 6000$ ) was employed during this work, and the polymer conjugates 1 were obtained from a four-step synthetic protocol reported by our group. ${ }^{14 e, 25}$ These substrates possess a built-in structural diversity (R1), from which a six-step sequence has been initiated by an intramolecular nucleophilic attack of the amino group on the amide carbonyl group. This ring closure reaction, followed by dehydration, was achieved using TFA (5\%) in 1,2-dichloroethane under refluxing conditions to lead the benzimidazole conjugates 2 . The addition of anhydrous magnesium sulfate in this transformation reduced the reaction time from 20 to $12 \mathrm{~h}$ by facilitation of the elimination of water during this step.

Magnesium sulfate was filtered off, and the polymer conjugate 2 precipitated and washed with excess of cold ether. The purity of this intermediate was excellent $\left({ }^{1} \mathrm{H}\right.$ NMR), and it was used directly for further steps in the present sequence. The reactivity of the ortho-fluoro nitro groups of $\mathbf{2}$ was explored in a cascaded manner with the introduction of second point of structural diversity (R2). The ipso-fluoro displacement with primary amines at room temperature in a typical $S_{N} A r$ reaction resulted in the polymer bound $o$-nitroanilines 3 . Generally, the transformation of the nitro group to an amino function requires either a mineral acid or an alkali. Both these conditions may induce a premature cleavage from the polymer support. For the selective reduction, without the cleavage from the support, neutral conditions using excess of zinc dust and ammonium formate in methanol were employed. The reaction was found to be complete in $30 \mathrm{~min}$ at room temperature, leading to the diamine $\mathbf{4}$, which is the precursor for the cyclic thioureide moiety. The progress of this reaction was monitored periodically by checking the proton NMR spectra of the crude reaction mixtures. Earlier we found that both thiophosgene and thiocarbonyl imidazole (TCI) can complete this cyclization. ${ }^{14,, 25}$ During the present investigation, we employed thiocarbonyl imidazole and triethylamine for one-pot cyclization in 1,2- dichloroethane, and the results were identical to those obtained by the use of thiophosgene. The reaction was found to occur at room temperature in $15 \mathrm{~h}$. To decrease the reaction time, refluxing conditions were applied in dichloromethane, which reduced the reaction time but gave rise to an unexpected byproduct in the form of the chloromethyl sulfide 5, which was confirmed when the polymer support was released to give the monomers $\mathbf{6}$. It is important to note that further alkylation occurring after the formation of the cyclic thiourea would necessarily give rise to either a bis- $\mathrm{N}-\mathrm{N}^{\prime}$-substitution or an $\mathrm{N}-\mathrm{S}$-substitution, resulting from the nucleophilic attacks of the ring nitrogen or that of the exocyclic sulfur. The ${ }^{1} \mathrm{H}$ NMR of this product was characterized by a singlet belonging to methylene protons at $5.46 \mathrm{ppm}$. The predicted carbon NMR chemical shift (43.2 ppm) for the respective carbon also agreed with this observation. Compounds 6 did not exhibit any signal in the range of $60 \mathrm{ppm}$, indicating the absence of the $\mathrm{N}-\mathrm{CH}_{2} \mathrm{Cl}$ group (Table 1). These predicted values support the S-chloromethylation very well. It is interesting to note that similar chloromethylation is observed in the enzymatic reaction of rat liver glutathione$\mathrm{S}$-transferases. The present reaction can be a nonenzymatic model for the biochemical process. ${ }^{26}$ Compounds isolated in considerable yields and purity are presented in Table 1 .

However, the reaction of diamines $\mathbf{4}$ with TCI at room temperature gave the desired polymer-bound bis-benzimidazolyl-2-thiones 7 when the solvent was changed from dichloromethane to 1,2-dichloroethane. This resulted in our first molecular library of benzimidazolyl benzimidazolin-2thiolnes 8, after cleavage, with two points of structural diversity (Table 2). To the best of our knowledge, it is the first report on this skeleton.

The third point of chemical diversity was introduced by the $\mathrm{N}$-alkylation of benzimidazolin-2-thione polymer conjugates 7. A survey in reactivity profile of benzimidazolin-2-thiones with carbon electrophiles indicates that use of formaldehyde and the corresponding Mannich reactions will lead to $\mathrm{N}-\mathrm{N}^{\prime}$-bis-alkylation. ${ }^{28,29}$ Under mild alkaline conditions, S-alkylation has been reported to occur and was employed to synthesize a number of biologically active sulfides, as well as to be kinetically investigated. ${ }^{30}$ The use of 1,3-dialkyl halides leads to tricyclic thiazinobenzimidazoles via $\mathrm{S}-\mathrm{N}$-bis-alkylation. ${ }^{31}$ In accordance with these reports, alkylation of $\mathbf{7}$ in principle can result in $\mathrm{N}$ - or S-alkylation. Under the room-temperature conditions employed, the reactions with various alkyl halides in presence of triethylamine led to S-alkylated conjugates 9. Similar cleavage of the polymer, under identical conditions was also marked by the disappearance of the strong signal around $4.2 \mathrm{ppm}$ caused by the $\mathrm{O}-\mathrm{CH}_{2}-\mathrm{PEG}$ with the appearance of a singlet around 3.9-4.0 ppm, which is caused by the $\mathrm{OCH}_{3}$ protons of the methyl ester. This resulted in the final 2-sulfanylated bis-benzimidazoles 10 (Table 3 and Scheme 2). In the proton NMR, the predicted chemical shifts for th $\mathrm{N}-\mathrm{CH}_{3}$ and $\mathrm{S}-\mathrm{CH}_{3}$ protons are 3.70 and $2.57 \mathrm{ppm}$, respectively. The chemical shift values in case of the compounds $\mathbf{1 0 a}-\mathbf{c}$ are in the 
Scheme 1. Synthetic Route for Bis-benzimidazolyl-2-thiones 8

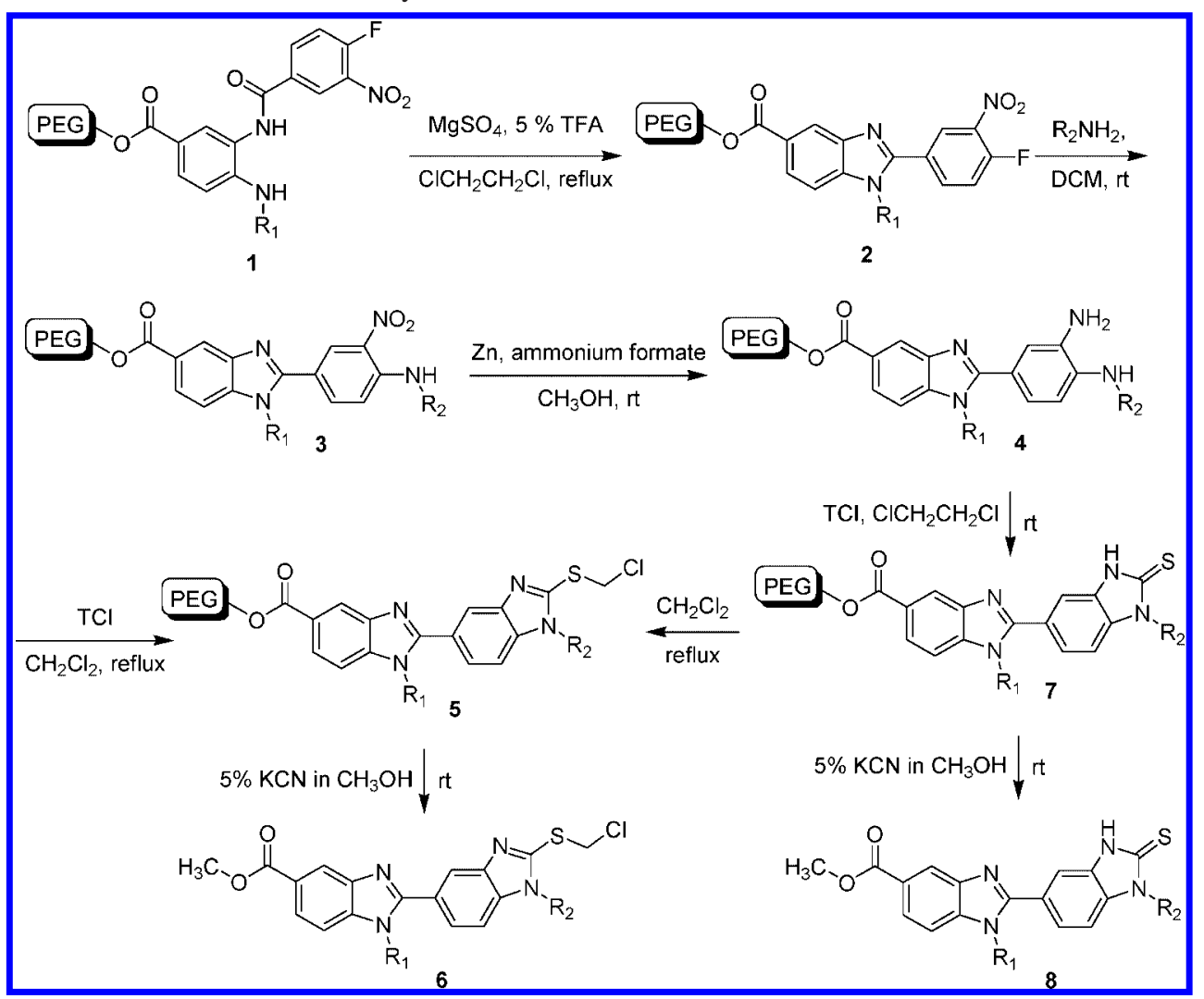

Table 1. Bis-benzimidazolyl Chloromethyl Sulphides 6

\begin{tabular}{|c|c|c|c|c|}
\hline Entry & $\mathrm{R}_{\mathbf{1}} \mathrm{NH}_{\mathbf{2}}$ & $\mathrm{R}_{2} \mathrm{NH}_{2}$ & LRMS $^{a}$ & $\begin{array}{c}\text { isolated } \\
\text { yield }(\%)^{b}\end{array}$ \\
\hline $6 a$ & & & 470 & 66 \\
\hline $6 b$ & & & 486 & 68 \\
\hline $6 c$ & & & 500 & 72 \\
\hline $6 d$ & & & 470 & 76 \\
\hline $6 e$ & & & $514(M+1)$ & 69 \\
\hline $6 f$ & & & $471(M+1)$ & 70 \\
\hline
\end{tabular}

${ }^{a}$ LRMS were detected with EI ionization source. ${ }^{b}$ Yields were based on loading of soluble support. range of $2.81-2.83 \mathrm{ppm}$, which also supports the observed regioselectivity. This twenty-member molecular library (Table 3) was obtained after seven steps, and all the final compounds have a high degree of purity (HPLC), so that they can be used directly in biological assays without any purification.

\section{Conclusions}

The hitherto described multistep sequence of reactions has led to the generation of molecular libraries with two core skeletons suitable for DNA minor groove recognition and enzyme susceptibility. This investigation also caused a model reaction that can mimic the bacterial enzymatic pathway. The site of alkylation has been proven by ${ }^{1} \mathrm{H}$ and ${ }^{13} \mathrm{C}$ NMR spectra. The introduction of the alkyl groups was carried out in an unambiguous manner without formation of any mixtures, and

Scheme 2. Liquid-Phase Synthesis of 2-Sulphanylated Bisbenzimidazoles

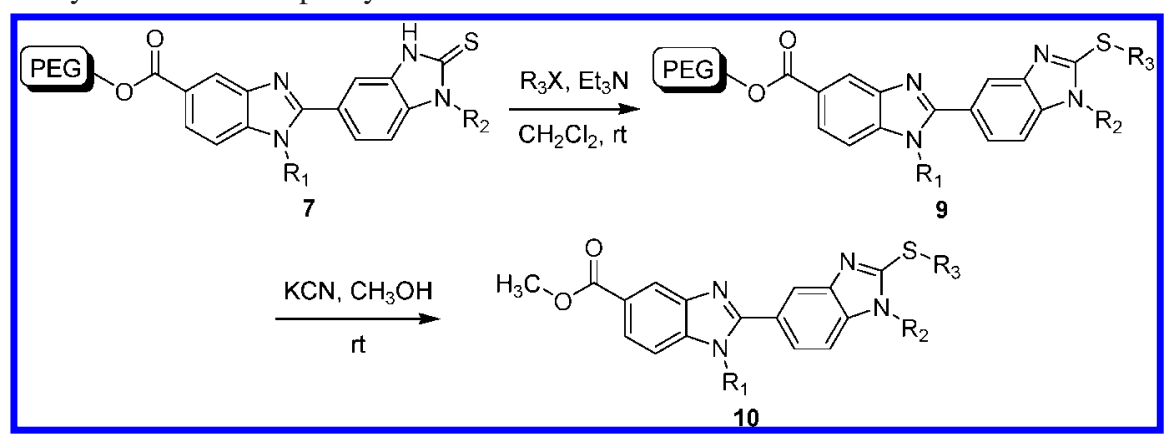


Table 2. Synthesis of Bis-benzimidazolyl-2-thiones $\mathbf{8}$

\begin{tabular}{|c|c|c|c|c|}
\hline Entry & $\mathrm{R}_{1} \mathrm{NH}_{2}$ & $\mathrm{R}_{2} \mathrm{NH}_{2}$ & LRMS $^{a}$ & $\begin{array}{c}\text { isolated } \\
\text { yield }(\%)^{b}\end{array}$ \\
\hline $8 a$ & & & 422 & 66 \\
\hline $8 \mathrm{~b}$ & & & 422 & 64 \\
\hline $8 c$ & & & 448 & 73 \\
\hline $8 d$ & & & 452 & 62 \\
\hline $8 \mathrm{e}$ & & & 474 & 73 \\
\hline $8 f$ & & & $499(M+1)$ & 66 \\
\hline $8 g$ & & & $491(M+1)$ & 79 \\
\hline $8 \mathrm{~h}$ & & & 462 & 81 \\
\hline $8 i$ & & & 476 & 86 \\
\hline
\end{tabular}

${ }^{a}$ LRMS were detected with EI ionization source. ${ }^{b}$ Yields were based on loading of soluble support.

all the final products have been obtained in high yields and purities by simple ether precipitation and washing.

\section{Experimental Section}

General Procedures for the Synthesis of Benzimidazolyl Benzimidazolones (8 and 10). To the solution of polymer immobilized conjugate $\mathbf{1}(0.33 \mathrm{mmol}, 1.0$ equiv) in 1,2-dichloroethane, trifluoroacetic acid $(0.5 \mathrm{~mL})$ and magnesium sulfate $(0.5 \mathrm{~g})$ were added. The mixture was refluxed for $12 \mathrm{~h}$, and the progress of the reaction was monitored by ${ }^{1} \mathrm{H}$ NMR. After completion of the reaction, $\mathrm{MgSO}_{4}$ was removed by filtration through a layer of Celite. Then the solvent was evaporated, and the product was precipitated by slow addition of an excess of cold ether. The precipitated benzimidazole conjugate $\mathbf{2}$ was filtered through a fritted funnel and washed with ether. The resulting conjugate $\mathbf{2}$ was treated with various amines ( $2.31 \mathrm{mmol}, 7.0$ equiv) in dichloromethane at ambient temperature for $12 \mathrm{~h}$. When the reaction was finished, the solvent was changed from dichloromethane to methanol because most of the excess amine that would hinder the zinc reduction can be removed by precipitation from methanol and because a small amount of conjugate $\mathbf{3}$ may be lost during ether washing. Conjugate $\mathbf{3}$ in methanol was completely reduced by zinc (3.3 mmol, 30.0 equiv) and ammonium formate $(3.75 \mathrm{mmol}, 15.0$ equiv) for $30 \mathrm{~min}$ at ambient temperature. Zinc was removed by centrifugation and filtration, and dichloromethane was added to precipitate ammonium formate. After filtration through Celite, polymer-immobilized diamine $\mathbf{4}$ was obtained. Polymer conjugate 4 ( $0.33 \mathrm{mmol}, 1.0$ equiv), triethylamine ( $0.78 \mathrm{mmol}, 2.6$ equiv), and thiocarbonyldiimidazole $(0.78 \mathrm{mmol}, 2.6$ equiv) were mixed in 1,2-dichloroethane, and the reaction mixtures were refluxed for $12 \mathrm{~h}$. The cyclyzed conjugated 7 was isolated by a similar ether precipitation and washing procedure. To the conjugated 7 ( $0.33 \mathrm{mmol}, 1.0$ equiv) in dichloromethane was added triethylamine (1.0 mmol, 3.3 equiv) and various alkyl halides ( $1.0 \mathrm{mmol}, 3.3$ equiv) at room temperature, and the reaction mixture was reacted for $14 \mathrm{~h}$ under ambient temperature. Conjugated 9 was purified by precipitation and washing with ether. The target compound was obtained by treating conjugate 9 with potassium cyanide in methanol for 3 days. The crude products $\mathbf{8}$ and $\mathbf{1 0}$ were obtained and subsequently checked by HPLC after precipitation. The crude products were then purified by column chromatography $\left(\mathrm{CH}_{2} \mathrm{Cl}_{2} / \mathrm{MeOH}=\right.$ 30:1 for $8 ; \mathrm{CH}_{2} \mathrm{Cl}_{2} / \mathrm{MeOH}=50: 1$ for 10 ), and the final products were obtained in high overall yield.

General procedures for the Synthesis of Benzimidazolyl Benzimidazolones (6). Polymer conjugate $4(0.33 \mathrm{mmol}, 1.0$ equiv), triethylamine ( $0.78 \mathrm{mmol}, 2.6$ equiv), and thiocarbonyldiimidazole $(0.78 \mathrm{mmol}, 2.6$ equiv) were mixed in dichloromethane, and the reaction mixture was refluxed for $12 \mathrm{~h}$. The cyclyzed conjugated $\mathbf{5}$ was isolated by a similar ether precipitation and washing procedure. Target compound $\mathbf{6}$ was obtained by treatment of conjugate $\mathbf{5}$ with potassium cyanide in methanol for $3 \mathrm{~h}$. Spectroscopic data are enclosed in the Supporting Information.

$1^{\prime}$-Isobutyl-1-isopropyl-2'-methylsulfanyl-1 $H, 1^{\prime} H$ [2,5']bibenzoimidazolyl-5-carboxylic Acid Methyl Ester (10a). ${ }^{1} \mathrm{H}$ NMR $\left(300 \mathrm{MHz}, \mathrm{CDCl}_{3}\right): \delta 8.52(\mathrm{~d}, J=1.1 \mathrm{~Hz}$, $1 \mathrm{H}), 8.00(\mathrm{dd}, J=8.5,1.5 \mathrm{~Hz}, 1 \mathrm{H}), 7.85(\mathrm{~d}, J=1.1 \mathrm{~Hz}$, $1 \mathrm{H}), 7.68(\mathrm{dd}, J=8.5,1.4 \mathrm{~Hz}, 1 \mathrm{H}), 7.58(\mathrm{~d}, J=8.5 \mathrm{~Hz}$, $1 \mathrm{H}), 7.44(\mathrm{~d}, J=8.5 \mathrm{~Hz}, 1 \mathrm{H}), 5.02-4.9(\mathrm{~m}, 1 \mathrm{H}), 3.95(\mathrm{~s}$, $3 \mathrm{H}), 3.97 \sim 3.91(\mathrm{~m}, 2 \mathrm{H}), 2.83(\mathrm{~s}, 3 \mathrm{H}), 2.25$ (septet, $J=7.0$ $\mathrm{Hz}, 1 \mathrm{H}), 1.65(\mathrm{~d}, J=6.9 \mathrm{~Hz}, 6 \mathrm{H}), 1.00(\mathrm{~d}, J=6.6 \mathrm{~Hz}$, $6 \mathrm{H}) .{ }^{13} \mathrm{C}$ NMR $\left(75 \mathrm{MHz}, \mathrm{CDCl}_{3}\right) \delta 167.7,156.0,155.4$, 143.2, 143.1, 138.0, 136.8, 124.2, 123.9, 123.7, 122.2, 118.7, 111.9, 109.7, 52.1, 51.8, 50.6, 49.1, 29.0, 21.5, 20.3, 14.8. IR (KBr): 2960, 1715, 1434, $1303 \mathrm{~cm}^{-1}$. EI-MS m/z $436\left(\mathrm{M}^{+}\right)$. HRMS $m / z$ calcd for $\mathrm{C}_{24} \mathrm{H}_{28} \mathrm{~N}_{4} \mathrm{O}_{2} \mathrm{~S}: 436.1929$. Found: 436.1933 .

$\mathbf{1}^{\prime}$-Butyl-1-isobutyl-2'-methylsulfanyl-1H,1'H-[2,5']bibenzoimidazolyl-5-carboxylic acid methyl ester (10b). ${ }^{1} \mathrm{H}$ NMR (300 MHz, $\left.\mathrm{CDCl}_{3}\right): \delta 8.50(\mathrm{~d}, J=1.1 \mathrm{~Hz}, 1 \mathrm{H}), 8.01$ (dd, $J=8.4,1.5 \mathrm{~Hz}, 1 \mathrm{H}), 7.89(\mathrm{~d}, J=1.2 \mathrm{~Hz}, 1 \mathrm{H}), 7.58$ $(\mathrm{dd}, J=8.5,1.5 \mathrm{~Hz}, 1 \mathrm{H}), 7.41-7.32(\mathrm{~m}, 2 \mathrm{H}), 4.16(\mathrm{~d}, J=$ $7.6 \mathrm{~Hz}, 2 \mathrm{H}), 4.10$ (t, $J=7.1 \mathrm{~Hz}, 2 \mathrm{H}), 3.93(\mathrm{~s}, 3 \mathrm{H}), 2.81$ (s, $3 \mathrm{H}), 2.18-2.00(\mathrm{~m}, 1 \mathrm{H}), 1.86-1.77(\mathrm{~m}, 2 \mathrm{H}), 1.45-1.34(\mathrm{~m}$ $2 \mathrm{H}), 0.94(\mathrm{t}, J=7.1 \mathrm{~Hz}, 3 \mathrm{H}), 0.67(\mathrm{~d}, J=6.6 \mathrm{~Hz}, 6 \mathrm{H}) .{ }^{13} \mathrm{C}$ NMR (75 MHz, $\left.\mathrm{CDCl}_{3}\right): \delta 167.7,156.7,154.7,143.4,142.7$, 139.2, 137.5, 124.3, 123.9, 123.7, 122.1, 118.8, 111.2, 109.2, 52.1, 52.0, 44.1, 31.3, 28.7, 20.1, 19.9, 14.6, 13.7. IR (KBr): 2958, 1714, 1435, $1301 \mathrm{~cm}^{-1}$. EI-MS $m / z: 450\left(\mathrm{M}^{+}\right)$HRMS $m / z$ calcd for $\mathrm{C}_{25} \mathrm{H}_{30} \mathrm{~N}_{4} \mathrm{O}_{2} \mathrm{~S}: 450.2089$. Found: 450.2092 .

1'-Butyl-1-(3-methoxy-propyl)-2'-methylsulfanyl- $1 \mathrm{H,1} \mathbf{1}^{\prime} \mathrm{H}$ [2,5']bibenzo-imidazolyl-5-carboxylic Acid Methyl Ester (10c). ${ }^{1} \mathrm{H}$ NMR $\left(300 \mathrm{MHz}, \mathrm{CDCl}_{3}\right): \delta 8.52(\mathrm{~d}, J=1.2 \mathrm{~Hz}$, $1 \mathrm{H}), 8.04(\mathrm{dd}, J=8.5,1.5 \mathrm{~Hz}, 1 \mathrm{H}), 7.96(\mathrm{~d}, J=1.1 \mathrm{~Hz}$, $1 \mathrm{H}), 7.65(\mathrm{dd}, J=8.3,1.5 \mathrm{~Hz}, 1 \mathrm{H}), 7.49(\mathrm{~d}, J=8.5 \mathrm{~Hz}$, $1 \mathrm{H}), 7.39$ (d, $J=8.3 \mathrm{~Hz}, 1 \mathrm{H}), 4.46(\mathrm{t}, J=7.0 \mathrm{~Hz}, 2 \mathrm{H})$, $4.41(\mathrm{~d}, J=7.2 \mathrm{~Hz}, 2 \mathrm{H}), 3.96(\mathrm{~s}, 3 \mathrm{H}), 3.22(\mathrm{t}, J=5.6 \mathrm{~Hz}$, $2 \mathrm{H}), 3.18(\mathrm{~s}, 3 \mathrm{H}), 2.83(\mathrm{~s}, 3 \mathrm{H}), 2.05-1.92(\mathrm{~m}, 2 \mathrm{H})$, $1.90-1.78(\mathrm{~m}, 2 \mathrm{H}), 1.55-1.30(\mathrm{~m}, 2 \mathrm{H}), 0.98(\mathrm{t}, J=7.2$ 
Table 3. 2-Sulphanylated Bis-benzimidazoles 10

\begin{tabular}{|c|c|c|c|c|c|c|}
\hline Entry & $\mathrm{R}_{1} \mathrm{NH}_{2}$ & $\mathrm{R}_{2} \mathrm{NH}_{2}$ & $\mathbf{R}_{\mathbf{3}}$ & LRMS $^{a}$ & $\begin{array}{l}\text { isolated } \\
\text { yield }(\%)^{b}\end{array}$ & $\begin{array}{l}\text { HPLC } \\
\text { purity }^{\circ}\end{array}$ \\
\hline $10 a$ & & & $\mathrm{CH}_{3} \mathrm{I}$ & 436 & 76 & 78 \\
\hline $10 \mathrm{~b}$ & & & $\mathrm{CH}_{3} \mathrm{I}$ & 450 & 78 & 85 \\
\hline $10 \mathrm{c}$ & & & $\mathrm{CH}_{3} \mathrm{I}$ & 466 & 80 & 76 \\
\hline $10 \mathrm{~d}$ & & & & 508 & 55 & 68 \\
\hline $10 \mathrm{e}$ & & & & 572 & 78 & 97 \\
\hline $10 f$ & & & $\mathrm{CH}_{3} \mathrm{I}$ & 488 & 81 & 94 \\
\hline $10 \mathrm{~g}$ & & & & 502 & 73 & 97 \\
\hline $10 \mathrm{~h}$ & & & & 594 & 76 & 89 \\
\hline $10 i$ & & & & 500 & 80 & 88 \\
\hline $10 \mathrm{j}$ & & & & 528 & 71 & 97 \\
\hline $10 \mathrm{k}$ & & & & 576 & 74 & 82 \\
\hline 101 & & & & 512 & 66 & 68 \\
\hline $10 \mathrm{~m}$ & & & & 524 & 78 & 92 \\
\hline $10 n$ & & & & 526 & 57 & 58 \\
\hline 100 & & & & 538 & 76 & 80 \\
\hline $10 p$ & & & & 526 & 71 & 94 \\
\hline $10 q$ & & & & 534 & 81 & 94 \\
\hline $10 r$ & & & & 582 & 80 & 90 \\
\hline $10 \mathrm{~s}$ & & & & 574 & 78 & 93 \\
\hline $10 t$ & & & & 630 & 68 & 82 \\
\hline
\end{tabular}

${ }^{a}$ LRMS were detected with EI ionization source. ${ }^{b}$ Yields were based on loading of soluble support. ${ }^{c}$ Crude products were analyzed by normalphase HPLC and monitored with a UV detector at a $254 \mathrm{~nm}$ wavelength (column silica, $250 \times 4.6 \mathrm{~mm}, 5 \mu \mathrm{m}$; eluent $5 \% \mathrm{MeOH} \mathrm{CH}_{2} \mathrm{Cl}_{2}$ ).

$\mathrm{Hz}, 3 \mathrm{H}) .{ }^{13} \mathrm{C} \mathrm{NMR}\left(75 \mathrm{MHz}, \mathrm{CDCl}_{3}\right): \delta 167.7,155.4,142.9$, $142.1,141.8,140.7,138.9,137.1,134.7,130.2,129.2,129.1$, $129.0,128.6,128.5,127.9,127.7,127.5,127.1,126.9,126.8$, 126.0, 124.0, 123.5, 122.2, 120.7, 119.1, 112.7, 111.9, 80.5, 59.0, 56.1, 53.3, 52.0, 44.9, 34.7, 33.7, 31.6, 30.0, 27.6, 27.5, 25.9, 25.8. IR (KBr): 2955, 1714, 1434, $1301 \mathrm{~cm}^{-1}$. EI-MS $m / z: 466\left(\mathrm{M}^{+}\right)$. HRMS m/z calcd for $\mathrm{C}_{25} \mathrm{H}_{30} \mathrm{~N}_{4} \mathrm{O}_{3} \mathrm{~S}: 466.2039$. Found: 466.2030.

2'-Butylsulfanyl-1'-isobutyl-1-(3-methoxy-propyl)- $1 H, 1^{\prime} H$ [2,5']bibenzo-imidazolyl-5-carboxylic Acid Methyl Ester (10d). ${ }^{1} \mathrm{H}$ NMR $\left(300 \mathrm{MHz}, \mathrm{CDCl}_{3}\right): \delta 8.50(\mathrm{~d}, J=1.2 \mathrm{~Hz}$, $1 \mathrm{H}), 8.02(\mathrm{dd}, J=8.5,1.5 \mathrm{~Hz}, 1 \mathrm{H}), 7.93(\mathrm{~d}, J=1.2 \mathrm{~Hz}$, 
1H), $7.62(\mathrm{dd}, J=8.3,1.5 \mathrm{~Hz}, 1 \mathrm{H}), 7.47(\mathrm{~d}, J=8.6 \mathrm{~Hz}$, $1 \mathrm{H}), 7.36(\mathrm{~d}, J=8.4 \mathrm{~Hz}, 1 \mathrm{H}), 4.45(\mathrm{t}, J=7.1 \mathrm{~Hz}, 2 \mathrm{H})$, 3.94 (s, 3H), 3.91 (d, $J=7.7 \mathrm{~Hz}, 2 \mathrm{H}), 3.42$ (t, $J=7.3 \mathrm{~Hz}$, $2 \mathrm{H}), 3.20(\mathrm{t}, J=5.6 \mathrm{~Hz}, 2 \mathrm{H}), 3.16(\mathrm{~s}, 3 \mathrm{H}), 2.40-2.20(\mathrm{~m}$, $2 \mathrm{H}), 2.03-1.94(\mathrm{~m}, 2 \mathrm{H}), 1.85-1.72(\mathrm{~m}, 2 \mathrm{H}), 1.55-1.46(\mathrm{~m}$, $2 \mathrm{H}), 0.97(\mathrm{~d}, J=6.7 \mathrm{~Hz}, 6 \mathrm{H}), 0.95(\mathrm{t}, J=7.4 \mathrm{~Hz}, 3 \mathrm{H}) .{ }^{13} \mathrm{C}$ NMR (75 MHz, $\left.\mathrm{CDCl}_{3}\right): \delta 167.7,149.3,149.0,142.2,138.4$, 135.0, 129.3, 127.0, 124.9, 123.3, 118.7, 114.6, 68.3, 52.0, 49.6, 44.0, 21.9, 20.0, 20.0. IR (KBr): 2957, 1715, 1433, $1301 \mathrm{~cm}^{-1}$. EI-MS $\mathrm{m} / \mathrm{z}: 508\left(\mathrm{M}^{+}\right)$. HRMS $\mathrm{m} / \mathrm{z}$ calcd for $\mathrm{C}_{28} \mathrm{H}_{36} \mathrm{~N}_{4} \mathrm{O}_{3} \mathrm{~S}$ : 508.2508. Found: 508.2500.

1'-Isobutyl-2'-(4-methoxy-benzylsulfanyl)-1-(3-methoxypropyl)-1H,1'H-[2,5']bibenzoimidazolyl-5-carboxylic Acid Methyl Ester (10e). ${ }^{1} \mathrm{H}$ NMR (300 MHz, $\left.\mathrm{CDCl}_{3}\right): \delta 8.52$ $(\mathrm{d}, J=1.3 \mathrm{~Hz}, 1 \mathrm{H}), 8.02(\mathrm{dd}, J=8.5,1.5 \mathrm{~Hz}, 1 \mathrm{H}), 7.98$ (d, $J=1.3 \mathrm{~Hz}, 1 \mathrm{H}), 7.64(\mathrm{dd}, J=8.3,1.5 \mathrm{~Hz}, 1 \mathrm{H}), 7.48(\mathrm{~d}, J$ $=8.6 \mathrm{~Hz}, 1 \mathrm{H}), 7.37(\mathrm{~d}, J=8.3 \mathrm{~Hz}, 1 \mathrm{H}), 7.22(\mathrm{t}, J=7.9$ $\mathrm{Hz}, 1 \mathrm{H}), 7.05-6.96(\mathrm{~m}, 2 \mathrm{H}), 6.80$ (dd, $J=8.8,1.8 \mathrm{~Hz}, 1 \mathrm{H})$, $4.61(\mathrm{~s}, 2 \mathrm{H}), 4.46$ (t, $J=6.9 \mathrm{~Hz}, 2 \mathrm{H}), 3.94$ (s, 3H), 3.87 (d, $J=7.6 \mathrm{~Hz}, 2 \mathrm{H}), 3.75(\mathrm{~s}, 3 \mathrm{H}), 3.21(\mathrm{t}, J=5.6 \mathrm{~Hz}, 2 \mathrm{H})$, $3.17(\mathrm{~s}, 3 \mathrm{H}), 2.30-2.15(\mathrm{~m}, 1 \mathrm{H}), 2.06-1.95(\mathrm{~m}, 2 \mathrm{H}), 0.93$ $(\mathrm{d}, J=6.7 \mathrm{~Hz}, 6 \mathrm{H}) .{ }^{13} \mathrm{C} \mathrm{NMR}\left(75 \mathrm{MHz}, \mathrm{CDCl}_{3}\right): \delta 202.6$, 166.9, 148.0, 147.8, 135.2, 131.8, 130.2, 129.0, 128.7, 128.5, 128.1, 123.7, 123.4, 122.0, 121.1, 120.4, 117.9, 117.7, 111.0, 58.0, 53.4, 51.7, 51.3, 28.0, 20.5. IR (KBr): 2957, 1714, $1434,1301 \mathrm{~cm}^{-1}$. EI-MS m/z: $572\left(\mathrm{M}^{+}\right)$. HRMS $\mathrm{m} / z$ calcd for $\mathrm{C}_{32} \mathrm{H}_{36} \mathrm{~N}_{4} \mathrm{O}_{4} \mathrm{~S}: 572.2457$. Found: 572.2465.

1-(2-Cyclohex-1-enyl-ethyl)-1'-isopropyl-2'-methylsulfanyl-1H, $\mathbf{1}^{\prime} H$-[2,5']bibenzoimidazolyl-5-carboxylic Acid Methyl Ester (10f). ${ }^{1} \mathrm{H}$ NMR (300 MHz, $\left.\mathrm{CDCl}_{3}\right): \delta 8.48$ $(\mathrm{s}, 1 \mathrm{H}), 7.97(\mathrm{~d}, J=8.5 \mathrm{~Hz}, 2 \mathrm{H}), 7.92(\mathrm{~s}, 1 \mathrm{H}), 7.54(\mathrm{q}, J=$ $8.5 \mathrm{~Hz}, 2 \mathrm{H}) 5.12(\mathrm{~s}, 1 \mathrm{H}), 4.66$ (septet, $J=6.9 \mathrm{~Hz}, 1 \mathrm{H}$ ), $3.90(\mathrm{~s}, 3 \mathrm{H}), 2.77$ (s, 3H), 2.29 (t, $J=6.8 \mathrm{~Hz}, 2 \mathrm{H})$, $1.84 \sim 1.62(\mathrm{~m}, 4 \mathrm{H}), 1.61(\mathrm{~d}, J=6.9 \mathrm{~Hz}, 6 \mathrm{H}), 1.42-1.30$ $(\mathrm{m}, 4 \mathrm{H}) .{ }^{13} \mathrm{C}$ NMR $\left(75 \mathrm{MHz}, \mathrm{CDCl}_{3}\right): \delta 167.8,156.1,142.8$, $142.7,142.3,142.2,138.9,133.2,129.1,128.5,128.5,128.4$, $127.9,127.7,127.2,125.8,124.7,124.4,124.1,122.1,120.8$, 117.4, 114.6, 109.9, 72.6, 52.1, 48.5, 43.8, 38.0, 33.5, 29.7, 28.2, 25.2, 22.7, 22.0. IR (KBr): 2929, 1714, 1435, 1294 $\mathrm{cm}^{-1}$. EI-MS $\mathrm{m} / \mathrm{z}: 488\left(\mathrm{M}^{+}\right)$. HRMS $\mathrm{m} / \mathrm{z}$ calcd for $\mathrm{C}_{28} \mathrm{H}_{32} \mathrm{~N}_{4} \mathrm{O}_{2} \mathrm{~S}$ : 488.2246. Found: 488.2251.

1-(2-Cyclohex-1-enyl-ethyl)-2'-ethylsulfanyl-1'-isopropyl-1H,1'H-[2,5']bibenzoimidazolyl-5-carboxylic Acid Methyl Ester (10g). ${ }^{1} \mathrm{H}$ NMR $\left(300 \mathrm{MHz}, \mathrm{CDCl}_{3}\right): \delta 8.50(\mathrm{~d}, J=$ $1.3 \mathrm{~Hz}, 1 \mathrm{H}), 8.01(\mathrm{dd}, J=8.5,1.5 \mathrm{~Hz}, 1 \mathrm{H}), 7.93(\mathrm{~s}, 1 \mathrm{H})$, $7.64-7.56(\mathrm{~m}, 2 \mathrm{H}), 7.41(\mathrm{~d}, J=8.5 \mathrm{~Hz}, 1 \mathrm{H}), 5.16(\mathrm{~s}, 1 \mathrm{H})$, 4.73 (septet, $J=6.9 \mathrm{~Hz}, 1 \mathrm{H}$ ), 4.39 (t, $J=7.1 \mathrm{~Hz}, 2 \mathrm{H}), 3.93$ (s, 3H), 3.39 (q, $J=7.4 \mathrm{~Hz}, 2 \mathrm{H}), 2.33(\mathrm{t}, J=7.1 \mathrm{~Hz}, 2 \mathrm{H})$, $1.84-1.66(\mathrm{~m}, 4 \mathrm{H}), 1.63(\mathrm{~d}, J=6.9 \mathrm{~Hz}, 6 \mathrm{H}), 1.46(\mathrm{t}, J=$ $7.4 \mathrm{~Hz}, 3 \mathrm{H}), 1.42-1.35(\mathrm{~m}, 4 \mathrm{H}) .{ }^{13} \mathrm{C}$ NMR $(75 \mathrm{MHz}$, $\left.\mathrm{CDCl}_{3}\right): \delta 156.1,145.1,144.6,143.3,142.7,138.9,136.6$, $134.2,133.2,130.8,129.2,128.9,128.7,128.5,128.45$, $127.9,127.7,126.2,126.1,126.05,125.8,125.0,124.9$, 124.7, 124.6, 124.4, 122.1, 120.3, 117.4, 109.9, 71.8, 52.1, 50.0, 48.5, 48.1, 43.9, 42.4, 38.0, 33.8, 31.6, 29.8, 28.2, 25.1, 22.7. IR (KBr): $2930,1715,1434,1293 \mathrm{~cm}^{-1}$. EI-MS m/z: $502\left(\mathrm{M}^{+}\right)$. HRMS $m / z$ calcd for $\mathrm{C}_{29} \mathrm{H}_{34} \mathrm{~N}_{4} \mathrm{O}_{2} \mathrm{~S}$ : 502.2402. Found: 502.2404.
1-(2-Cyclohex-1-enyl-ethyl)-1'-isopropyl-2' -(4-methoxybenzylsulfanyl)-1 $H, 1^{\prime} H$-[2,5']bibenzoimidazolyl-5-carboxylic Acid Methyl Ester (10h). ${ }^{1} \mathrm{H}$ NMR (300 MHz, $\left.\mathrm{CDCl}_{3}\right): \delta 8.52(\mathrm{~d}, J=1.3 \mathrm{~Hz}, 1 \mathrm{H}), 8.03(\mathrm{dd}, J=8.5,1.5$ $\mathrm{Hz}, 1 \mathrm{H}), 7.98(\mathrm{~d}, J=1.1 \mathrm{~Hz}, 1 \mathrm{H}), 7.61(\mathrm{dd}, J=8.5,1.5$ $\mathrm{Hz}, 1 \mathrm{H}), 7.56(\mathrm{~d}, J=8.4 \mathrm{~Hz}, 1 \mathrm{H}), 7.42(\mathrm{~d}, J=8.5 \mathrm{~Hz}$, $1 \mathrm{H}), 7.21$ (t, $J=7.8 \mathrm{~Hz}, 1 \mathrm{H}), 6.99(\mathrm{~d}, J=9.6 \mathrm{~Hz}, 2 \mathrm{H})$, $6.80(\mathrm{~d}, J=8.1 \mathrm{~Hz}, 1 \mathrm{H}), 5.19$ (s, $1 \mathrm{H}), 4.71$ (septet, $J=6.9$ $\mathrm{Hz}, 1 \mathrm{H}), 4.60$ (s, 2H), $4.41(\mathrm{~d}, J=6.9 \mathrm{~Hz}, 2 \mathrm{H}), 3.95$ (s, $3 \mathrm{H}), 3.75(\mathrm{~s}, 3 \mathrm{H}), 2.36(\mathrm{t}, J=7.0 \mathrm{~Hz}, 2 \mathrm{H}), 1.84-1.70(\mathrm{~m}$, $4 \mathrm{H}), 1.59(\mathrm{~d}, J=6.9 \mathrm{~Hz}, 6 \mathrm{H}), 1.48-1.30(\mathrm{~m}, 4 \mathrm{H}) .{ }^{13} \mathrm{C} \mathrm{NMR}$ (75 MHz, $\left.\mathrm{CDCl}_{3}\right): \delta 167.7,161.9,158.6,155.9,143.0,142.6$, $138.9,138.5129 .8,129.7,129.2,129.2,129.0,128.5,128.3$ $127.9,127.7,127.3,124.4,124.2,123.9,123,8,122.1,120.3$, 117.7, 116.0, 115.7, 114.5, 114.4, 109.8, 96.0, 88.1, 70.0, 67.0, 57.0, 52.1, 47.7, 44.8, 31.9, 29.7, 20.0, 15.6, 13.6. IR (KBr): 2932, 1714, 1434, $1294 \mathrm{~cm}^{-1}$. ESI-MS m/z: 595 (M $+1)$. HRMS $m / z$ calcd for $\mathrm{C}_{35} \mathrm{H}_{38} \mathrm{~N}_{4} \mathrm{O}_{3} \mathrm{~S}: 594.2665$. Found: 594.2659.

2'-Allylsulfanyl-1-butyl-1'-furan-2-ylmethyl-1H,1'H[2,5']bibenzoimid-azolyl-5-carboxylic Acid Methyl Ester (10i). ${ }^{1} \mathrm{H}$ NMR $\left(300 \mathrm{MHz}, \mathrm{CDCl}_{3}\right): \delta 8.52(\mathrm{~d}, J=1.1 \mathrm{~Hz}$, $1 \mathrm{H}), 8.04(\mathrm{dd}, J=8.5,1.5 \mathrm{~Hz}, 1 \mathrm{H}), 7.93(\mathrm{~d}, J=1.1 \mathrm{~Hz}$, $1 \mathrm{H}), 7.64(\mathrm{dd}, J=8.5,1.5 \mathrm{~Hz}, 1 \mathrm{H}), 7.52(\mathrm{~d}, J=8.5 \mathrm{~Hz}$, $1 \mathrm{H}), 7.43(\mathrm{~d}, J=8.5 \mathrm{~Hz}, 1 \mathrm{H}), 7.36(\mathrm{~s}, 1 \mathrm{H}), 6.40 \sim 6.28(\mathrm{~m}$, 2H), $6.12-5.96(\mathrm{~m}, 1 \mathrm{H}), 5.35(\mathrm{~d}, J=18.3 \mathrm{~Hz}, 1 \mathrm{H}), 5.32(\mathrm{~s}$, $2 \mathrm{H}), 5.12(\mathrm{~d}, J=9.9 \mathrm{~Hz}, 1 \mathrm{H}), 4.29$ (t, $J=7.5 \mathrm{~Hz}, 2 \mathrm{H})$, 4.07 (d, $J=7.0 \mathrm{~Hz}, 2 \mathrm{H}), 3.95(\mathrm{~s}, 3 \mathrm{H}), 1.89-1.76(\mathrm{~m}, 2 \mathrm{H})$, $1.27-1.21(\mathrm{~m}, 2 \mathrm{H}), 0.83(\mathrm{t}, J=7.3 \mathrm{~Hz}, 3 \mathrm{H}) .{ }^{13} \mathrm{C}$ NMR $(75$ $\left.\mathrm{MHz}, \mathrm{CDCl}_{3}\right): \delta 167.7,156.0,142.9,142.6,140.8$ 139.0, 138.6, 133.5, 129.5, 129.3, 128.8, 128.3, 128.0, 127.3, 127.1, 124.4, 124.2, 123.5, 122.1, 120.0, 117.6, 114.2, 109.8, 73.2, 57.0, 52.1, 45.6, 44.7, 31.8, 22.7, 19.9, 13.6. IR (KBr): 2956, 1713, 1435, $1302 \mathrm{~cm}^{-1}$. ESI-MS m/z: $501(\mathrm{M}+1)$. HRMS $m / z$ calcd for $\mathrm{C}_{28} \mathrm{H}_{28} \mathrm{~N}_{4} \mathrm{O}_{3} \mathrm{~S}$ : 500.1882. Found: 500.1888 .

1-Butyl-1'-furan-2-ylmethyl-2' -(3-methyl-but-2-enylsulfanyl)-1H, $\mathbf{1}^{\prime} H$-[2,5']bibenzoimidazolyl-5-carboxylic Acid Methyl Ester (10j). ${ }^{1} \mathrm{H}$ NMR (300 MHz, $\left.\mathrm{CDCl}_{3}\right): \delta 8.52$ $(\mathrm{d}, J=1.1 \mathrm{~Hz}, 1 \mathrm{H}), 8.04(\mathrm{dd}, J=8.5,1.5 \mathrm{~Hz}, 1 \mathrm{H}), 7.93(\mathrm{~d}$, $J=1.1 \mathrm{~Hz}, 1 \mathrm{H}), 7.64(\mathrm{dd}, J=8.5,1.5 \mathrm{~Hz}, 1 \mathrm{H}), 7.52(\mathrm{~d}, J$ $=8.5 \mathrm{~Hz}, 1 \mathrm{H}), 7.43(\mathrm{~d}, J=8.5 \mathrm{~Hz}, 1 \mathrm{H}), 7.36(\mathrm{~s}, 1 \mathrm{H})$, $6.38-6.32(\mathrm{~m}, 2 \mathrm{H}), 5.43(\mathrm{t}, J=7.9 \mathrm{~Hz}, 1 \mathrm{H}), 5.30(\mathrm{~s}, 2 \mathrm{H})$, $4.29(\mathrm{t}, J=7.5 \mathrm{~Hz}, 2 \mathrm{H}), 4.07(\mathrm{~d}, J=7.9 \mathrm{~Hz}, 2 \mathrm{H}), 3.95$ (s, $3 \mathrm{H}), 1.84-1.70(\mathrm{~m}, 8 \mathrm{H}), 1.28-1.21(\mathrm{~m}, 2 \mathrm{H}), 0.83(\mathrm{t}, J=$ $7.3 \mathrm{~Hz}, 3 \mathrm{H}) .{ }^{13} \mathrm{C}$ NMR $\left(75 \mathrm{MHz}, \mathrm{CDCl}_{3}\right): \delta 167.8,161.5$, 158.2, 156.1, 143.6, 142.6, 138.9, 130.6, 130.4, 129.4, 129.3, $129.0,1288,128.7,126.9,124.3,124.0,123.7,123.6,122.1$, $121.7,117.2,115.9,115.6,114.0,109.8,64.1,59.1,52.1$, 44.6, 43.7, 31.8, 31.7, 31.6, 30.7, 29.7, 26.3, 25.7, 22.7, 19.9, 14.2. IR (KBr): $2955,1713,1435,1302 \mathrm{~cm}^{-1}$. EI-MS m/z: $528\left(\mathrm{M}^{+}\right)$. HRMS $\mathrm{m} / z$ calcd for $\mathrm{C}_{30} \mathrm{H}_{32} \mathrm{~N}_{4} \mathrm{O}_{3} \mathrm{~S}$ : 528.2195. Found: 528.2200.

Butyl-1'-furan-2-ylmethyl-2' -(3-phenyl-allylsulfanyl)$1 H, 1^{\prime} H$-[2,5']bibenzoimidazolyl-5-carboxylic Acid Methyl Ester (10k). ${ }^{1} \mathrm{H}$ NMR (300 MHz, $\left.\mathrm{CDCl}_{3}\right): \delta 8.53(\mathrm{~d}, J=$ $1.1 \mathrm{~Hz}, 1 \mathrm{H}), 8.04(\mathrm{dd}, J=8.5,1.5 \mathrm{~Hz}, 1 \mathrm{H}), 7.96(\mathrm{~d}, J=$ $1.1 \mathrm{~Hz}, 1 \mathrm{H}), 7.64(\mathrm{dd}, J=8.5,1.5 \mathrm{~Hz}, 1 \mathrm{H}), 7.52(\mathrm{~d}, J=$ $8.5 \mathrm{~Hz}, 1 \mathrm{H}), 7.44(\mathrm{~d}, J=8.5 \mathrm{~Hz}, 1 \mathrm{H}), 7.36-7.22(\mathrm{~m}, 6 \mathrm{H})$, $6.67(\mathrm{~d}, J=15.6 \mathrm{~Hz}, 1 \mathrm{H}), 6.48-6.28(\mathrm{~m}, 3 \mathrm{H}), 5.30(\mathrm{~s}, 2 \mathrm{H})$, 
$4.30(\mathrm{t}, J=7.5 \mathrm{~Hz}, 2 \mathrm{H}), 4.25(\mathrm{dd}, J=7.4,1.0 \mathrm{~Hz}, 2 \mathrm{H})$, $3.94(\mathrm{~s}, 3 \mathrm{H}), 1.86-1.72(\mathrm{~m}, 2 \mathrm{H}), 1.32-1.20(\mathrm{~m}, 2 \mathrm{H}), 0.84$ $(\mathrm{t}, J=7.3 \mathrm{~Hz}, 3 \mathrm{H}) .{ }^{13} \mathrm{C}$ NMR $\left(75 \mathrm{MHz}, \mathrm{CDCl}_{3}\right): \delta 167.7$, 156.1, 143.6, 142.7, 141.7, 138.9, 133.5, 129.2, 129.0, 127.1, 124.4, 124.1, 122.8, 122.2, 122.1, 117.2, 114.0, 109.8, 60.4, 53.5, 52.1, 44.6, 43.0, 31.8, 31.6, 31.1, 26.2, 26.0, 25.9, 19.9, 13.5. IR (KBr): $2955,1713,1435,1301 \mathrm{~cm}^{-1}$. ESI-MS m/z: $577(\mathrm{M}+1)$. HRMS $m / z$ calcd for $\mathrm{C}_{34} \mathrm{H}_{32} \mathrm{~N}_{4} \mathrm{O}_{3} \mathrm{~S}:$ 576.2195. Found: 576.2200.

2'-Ethylsulfanyl-1'-isopropyl-1-(3-phenyl-propyl)-1H, $1^{\prime} H$ [2,5']bibenzo-imidazolyl-5-carboxylic Acid Methyl Ester (101). ${ }^{1} \mathrm{H}$ NMR $\left(300 \mathrm{MHz}, \mathrm{CDCl}_{3}\right): \delta 8.55(\mathrm{~d}, J=1.1 \mathrm{~Hz}$, $1 \mathrm{H}), 8.02(\mathrm{dd}, J=8.5,1.5 \mathrm{~Hz}, 1 \mathrm{H}), 7.94(\mathrm{~s}, 1 \mathrm{H}), 7.54(\mathrm{~s}$, $2 \mathrm{H}), 7.29(\mathrm{~d}, J=8.4 \mathrm{~Hz}, 1 \mathrm{H}), 7.26-7.12(\mathrm{~m}, 3 \mathrm{H}), 7.04(\mathrm{~d}$, $J=6.5 \mathrm{~Hz}, 2 \mathrm{H}), 4.77$ (septet, $J=6.9 \mathrm{~Hz}, 1 \mathrm{H}), 4.33(\mathrm{t}, J=$ $7.5 \mathrm{~Hz}, 2 \mathrm{H}), 3.96(\mathrm{~s}, 3 \mathrm{H}), 3.44(\mathrm{q}, J=7.4 \mathrm{~Hz}, 2 \mathrm{H}), 2.56(\mathrm{t}$, $J=7.4 \mathrm{~Hz}, 2 \mathrm{H}), 2.18 \sim 2.08(\mathrm{~m}, 2 \mathrm{H}), 1.68(\mathrm{~d}, J=6.9 \mathrm{~Hz}$, $6 \mathrm{H}), 1.51(\mathrm{t}, J=7.4 \mathrm{~Hz}, 3 \mathrm{H}) .{ }^{13} \mathrm{C}$ NMR $\left(75 \mathrm{MHz}, \mathrm{CDCl}_{3}\right)$ : $\delta 167.8,156.1,149.3,143.7,142.7,138.9,136.7,129.0$, $126.7,124.3,124.0,123.0,122.5,122.0,121.7,117.7,115.2$, $109.8,71.2,59.0,52.1,44.7,44.6,31.8,31.2,26.0,25.8$, 19.9, 13.6. IR (KBr): 2978, 1714, 1434, $1294 \mathrm{~cm}^{-1}$. ESIMS $m / z: 513(\mathrm{M}+1)$. HRMS m/z calcd for $\mathrm{C}_{30} \mathrm{H}_{32} \mathrm{~N}_{4} \mathrm{O}_{2} \mathrm{~S}$ : 512.2246. Found: 512.2201.

2'-Allylsulfanyl-1'-isopropyl-1-(3-phenyl-propyl)- $1 H, 1^{\prime} H$ [2,5']bibenzo-imidazolyl-5-carboxylic Acid Methyl Ester (10m). ${ }^{1} \mathrm{H}$ NMR $\left(300 \mathrm{MHz}, \mathrm{CDCl}_{3}\right): \delta 8.53(\mathrm{~d}, J=1.1 \mathrm{~Hz}$, $1 \mathrm{H}), 8.01(\mathrm{dd}, J=8.5,1.5 \mathrm{~Hz}, 1 \mathrm{H}), 7.93(\mathrm{~s}, 1 \mathrm{H}), 7.53(\mathrm{~s}$, $2 \mathrm{H}), 7.27(\mathrm{~d}, J=8.6 \mathrm{~Hz}, 1 \mathrm{H}), 7.25-7.13(\mathrm{~m}, 3 \mathrm{H}), 7.03(\mathrm{~d}$, $J=6.5 \mathrm{~Hz}, 2 \mathrm{H}), 6.14-6.09(\mathrm{~m}, 1 \mathrm{H}), 5.37(\mathrm{dd}, J=16.8$, $1.3 \mathrm{~Hz}, 1 \mathrm{H}$ ), 5.19 (dd, $J=10.0,1.3 \mathrm{~Hz}, 1 \mathrm{H}$ ), 4.80 (septet, $J=6.9 \mathrm{~Hz}, 1 \mathrm{H}), 4.32(\mathrm{t}, J=7.5 \mathrm{~Hz}, 2 \mathrm{H}), 4.07(\mathrm{~d}, J=6.1$ $\mathrm{Hz}, 2 \mathrm{H}), 3.95$ (s, 3H), 2.56 (t, $J=7.4 \mathrm{~Hz}, 2 \mathrm{H}), 2.20-2.09$ $(\mathrm{m}, 2 \mathrm{H}), 1.67(\mathrm{~d}, J=6.9 \mathrm{~Hz}, 6 \mathrm{H}) .{ }^{13} \mathrm{C}$ NMR $(75 \mathrm{MHz}$, $\left.\mathrm{CDCl}_{3}\right): \delta 202.6,166.9,148.0,147.8,135.2,131.8,130.2,129.0$, 128.7, 128.5, 128.1, 123.7, 123.4, 122.0, 121.1, 120.4, 117.9, 117.7, 111.0, 58.0, 53.4, 51.7, 51.3, 28.0, 20.5. IR (KBr): 2946, 1714, 1434, $1294 \mathrm{~cm}^{-1}$. ESI-MS m/z: $525(\mathrm{M}+1)$. HRMS $m / z$ calcd for $\mathrm{C}_{31} \mathrm{H}_{32} \mathrm{~N}_{4} \mathrm{O}_{2} \mathrm{~S}$ : 524.2246. Found: 524.2238.

1'-Isopropyl-1-(3-phenyl-propyl)-2'-propylsulfanyl$1 H, 1^{\prime} H$-[2,5']bibenzo-imidazolyl-5-carboxylic Acid Methyl Ester (10n). ${ }^{1} \mathrm{H}$ NMR $\left(300 \mathrm{MHz}, \mathrm{CDCl}_{3}\right): \delta 8.55(\mathrm{~d}, J=$ $1.1 \mathrm{~Hz}, 1 \mathrm{H}), 8.02(\mathrm{dd}, J=8.5,1.5 \mathrm{~Hz}, 1 \mathrm{H}), 7.94(\mathrm{~s}, 1 \mathrm{H})$, $7.55(\mathrm{~s}, 2 \mathrm{H}), 7.29(\mathrm{~d}, J=8.4 \mathrm{~Hz}, 1 \mathrm{H}), 7.26-7.12(\mathrm{~m}, 3 \mathrm{H})$, $7.05(\mathrm{~d}, J=6.5 \mathrm{~Hz}, 2 \mathrm{H}), 4.80$ (septet, $J=6.9 \mathrm{~Hz}, 1 \mathrm{H})$ ), $4.34(\mathrm{t}, J=7.5 \mathrm{~Hz}, 2 \mathrm{H}), 3.97(\mathrm{~s}, 3 \mathrm{H}), 3.43(\mathrm{t}, J=7.2 \mathrm{~Hz}$, $2 \mathrm{H}), 2.57(\mathrm{t}, J=7.4 \mathrm{~Hz}, 2 \mathrm{H}), 2.19 \sim 2.10(\mathrm{~m}, 2 \mathrm{H}), 1.94-1.82$ $(\mathrm{m}, 2 \mathrm{H}), 1.69(\mathrm{~d}, J=6.9 \mathrm{~Hz}, 6 \mathrm{H}), 1.12(\mathrm{t}, J=7.3 \mathrm{~Hz}, 3 \mathrm{H})$. ${ }^{13} \mathrm{C}$ NMR $\left(75 \mathrm{MHz}, \mathrm{CDCl}_{3}\right): \delta 167.7,159.3,156.6,145.0$, 144.8, 143.4, 143.0, 136.6, 133.9, 129,4, 128.5, 128.4, 127.9, $127.8,127.8,127.5,126.2,126.1,124.1,123.5,122.4,121.0$, 117.7, 114.7, 113.8, 111.4, 73.0, 57.6, 55.3, 52.0, 50.1, 49.1, 48.5, 45.2, 45.1, 33.9, 30.4, 25.3. IR (KBr): 2948, 1714, 1434, $1293 \mathrm{~cm}^{-1}$. ESI-MS $m / z: 527(\mathrm{M}+1)$. HRMS $\mathrm{m} / \mathrm{z}$ calcd for $\mathrm{C}_{31} \mathrm{H}_{34} \mathrm{~N}_{4} \mathrm{O}_{2} \mathrm{~S}$ : 526.2402. Found: 526.2422.

2'-Allylsulfanyl-1-butyl-1'-(3-phenyl-propyl)-1H, 1' $H$ [2,5']bibenzo-imidazolyl-5-carboxylic Acid Methyl Ester (10o). ${ }^{1} \mathrm{H}$ NMR $\left(300 \mathrm{MHz}, \mathrm{CDCl}_{3}\right): \delta 8.54(\mathrm{~d}, J=1.2$ $\mathrm{Hz}, 1 \mathrm{H}), 8.05(\mathrm{dd}, J=8.5,1.5 \mathrm{~Hz}, 1 \mathrm{H}), 7.95(\mathrm{~d}, J=1.2$
$\mathrm{Hz}, 1 \mathrm{H}), 7.62(\mathrm{dd}, J=8.3,1.5 \mathrm{~Hz}, 1 \mathrm{H}), 7.44(\mathrm{~d}, J=8.6$ $\mathrm{Hz}, 1 \mathrm{H}), 7.38-7.16(\mathrm{~m}, 6 \mathrm{H}), 6.14-5.94(\mathrm{~m}, 1 \mathrm{H}), 5.38$ $(\mathrm{dd}, J=16.9,1.0 \mathrm{~Hz}, 1 \mathrm{H}), 5.20(\mathrm{dd}, J=9.9,1.0 \mathrm{~Hz}$, $1 \mathrm{H}), 4.32(\mathrm{t}, J=7.5,2 \mathrm{H}), 4.18(\mathrm{t}, J=7.4 \mathrm{~Hz}, 2 \mathrm{H}), 4.09$ (d. $J=7.0 \mathrm{~Hz}, 2 \mathrm{H}), 3.97(\mathrm{~s}, 3 \mathrm{H}), 2.74(\mathrm{t}, J=7.5 \mathrm{~Hz}$, $2 \mathrm{H}), 2.27-2.16(\mathrm{~m}, 2 \mathrm{H}), 1.91-1.79(\mathrm{~m}, 2 \mathrm{H}), 1.30-1.21$ $(\mathrm{m}, 2 \mathrm{H}), 0.85(\mathrm{t}, J=7.3 \mathrm{~Hz}, 3 \mathrm{H}) .{ }^{13} \mathrm{C}$ NMR $(75 \mathrm{MHz}$, $\left.\mathrm{CDCl}_{3}\right): \delta 167.7,156.0,143.4,143.0,142.5,136.9,129.3$, $128.4,127.8,127.6,127.3,124.0,123.6,122.3,121.3$, 117.5, 114.6, 111.8, 73.2, 60.7, 52.0, 49.0, 48.9, 26.6, 21.5, 20.8, 20.8. IR (KBr): 2956, 1715, 1434, $1301 \mathrm{~cm}^{-1}$. ESIMS $m / z: 539(\mathrm{M}+1)$. HRMS $m / z$ calcd for $\mathrm{C}_{32} \mathrm{H}_{34} \mathrm{~N}_{4} \mathrm{O}_{2} \mathrm{~S}$ : 538.2402. Found: 538.2439.

1-Butyl-2'-ethylsulfanyl-1' -(3-phenyl-propyl)-1 $H, 1^{\prime} H$ [2,5']bibenzo-imidazolyl-5-carboxylic Acid Methyl Ester (10p). ${ }^{1} \mathrm{H}$ NMR $\left(300 \mathrm{MHz}, \mathrm{CDCl}_{3}\right): \delta 8.52(\mathrm{~d}, J=1.2$ $\mathrm{Hz}, 1 \mathrm{H}), 8.04(\mathrm{dd}, J=8.5,1.5 \mathrm{~Hz}, 1 \mathrm{H}), 7.92(\mathrm{~d}, J=1.1$ $\mathrm{Hz}, 1 \mathrm{H}), 7.60(\mathrm{dd}, J=8.3,1.5 \mathrm{~Hz}, 1 \mathrm{H}), 7.34-7.16(\mathrm{~m}$, $6 \mathrm{H}), 4.31(\mathrm{t}, J=7.5 \mathrm{~Hz}, 2 \mathrm{H}), 4.16(\mathrm{t}, J=7.4 \mathrm{~Hz}, 2 \mathrm{H})$, $3.96(\mathrm{~s}, 3 \mathrm{H}), 3.43(\mathrm{q}, J=7.4 \mathrm{~Hz}, 2 \mathrm{H}), 2.73(\mathrm{t}, J=7.9$ $\mathrm{Hz}, 2 \mathrm{H}), 2.27-2.16(\mathrm{~m}, 2 \mathrm{H}), 1.83-1.74(\mathrm{~m}, 2 \mathrm{H}), 1.49(\mathrm{t}$, $J=7.4 \mathrm{~Hz}, 3 \mathrm{H}), 1.30-1.21(\mathrm{~m}, 2 \mathrm{H}), 0.83(\mathrm{t}, J=7.3 \mathrm{~Hz}$, $3 \mathrm{H}) .{ }^{13} \mathrm{C}$ NMR $\left(75 \mathrm{MHz}, \mathrm{CDCl}_{3}\right): \delta 167.7,159.1,156.0$, $143.4,143.0,142.5,136.9,129.3,128.4,127.8,127.6$, $127.3,124.0,123.6,122.3,121.3,117.5,114.6,111.8$, 73.2, 60.7, 52.0, 49.0, 48.9, 26.6, 21.5, 20.8, 20.8; IR (KBr): 2954, 1714, 1434, $1301 \mathrm{~cm}^{-1}$. ESI-MS m/z: 527 $(\mathrm{M}+1)$. HRMS $\mathrm{m} / z$ calcd for $\mathrm{C}_{31} \mathrm{H}_{34} \mathrm{~N}_{4} \mathrm{O}_{2} \mathrm{~S}: 526.2402$. Found: 526.2384.

1-(3-Methoxy-propyl)-2'-(3-methyl-but-2-enylsulfanyl)1'-(3-methyl-butyl)-1H,1'H-[2,5']bibenzoimidazolyl-5-carboxylic Acid Methyl Ester (10q). ${ }^{1} \mathrm{H}$ NMR (300 MHz, $\left.\mathrm{CDCl}_{3}\right): \delta 8.51(\mathrm{~d}, J=1.1 \mathrm{~Hz}, 1 \mathrm{H}), 8.02(\mathrm{dd}, J=8.5$, $1.5 \mathrm{~Hz}, 1 \mathrm{H}), 7.94(\mathrm{~d}, J=1.1 \mathrm{~Hz}, 1 \mathrm{H}), 7.63(\mathrm{dd}, J=8.3$, $1.5 \mathrm{~Hz}, 1 \mathrm{H}), 7.47(\mathrm{~d}, J=8.5 \mathrm{~Hz}, 1 \mathrm{H}), 7.37(\mathrm{~d}, J=8.3$ $\mathrm{Hz}, 1 \mathrm{H}), 5.42(\mathrm{t}, J=8.0 \mathrm{~Hz}, 1 \mathrm{H}), 4.44(\mathrm{t}, J=7.2 \mathrm{~Hz}$, $2 \mathrm{H}), 4.11(\mathrm{t}, J=7.5 \mathrm{~Hz}, 2 \mathrm{H}), 4.06(\mathrm{~d}, J=8.0 \mathrm{~Hz}, 2 \mathrm{H})$, $4.00(\mathrm{~s}, 3 \mathrm{H}), 3.21(\mathrm{t}, J=5.7 \mathrm{~Hz}, 2 \mathrm{H}), 3.17(\mathrm{~s}, 3 \mathrm{H})$, $2.04-1.95(\mathrm{~m}, 2 \mathrm{H}), 1.74(\mathrm{~s}, 6 \mathrm{H}), 1.66-1.60(\mathrm{~m}, 3 \mathrm{H}), 1.00$ $(\mathrm{d}, J=6.2 \mathrm{~Hz}, 6 \mathrm{H}) .{ }^{13} \mathrm{C}$ NMR $\left(75 \mathrm{MHz}, \mathrm{CDCl}_{3}\right): \delta 167.7$, $159.1,156.0,143.4,143.0,142.5,136.9,129.3,128.4$, $127.8,127.6,127.3,124.0,123.6,122.3,121.3,117.5$, 114.6, 111.8, 73.2, 60.7, 52.0, 49.0, 48.9, 26.6, 21.5, 20.8, 20.8. IR(KBr): $2954,1715,1433,1301 \mathrm{~cm}^{-1}$. ESI-MS $\mathrm{m} / \mathrm{z}: 535(\mathrm{M}+1)$. HRMS $\mathrm{m} / \mathrm{z}$ calcd for $\mathrm{C}_{30} \mathrm{H}_{38} \mathrm{~N}_{4} \mathrm{O}_{3} \mathrm{~S}$ : 534.2665. Found: 534.2665.

1-(3-Methoxy-propyl)-1'-(3-methyl-butyl)-2'-(3-phenylallylsulfanyl)-1 $H, 1^{\prime} H$-[2,5']bibenzoimidazolyl-5-carboxylic Acid Methyl Ester (10r). ${ }^{1} \mathrm{H}$ NMR (300 $\left.\mathrm{MHz}, \mathrm{CDCl}_{3}\right)$ : $\delta 8.53(\mathrm{~d}, J=1.2 \mathrm{~Hz}, 1 \mathrm{H}), 8.04(\mathrm{dd}, J=8.5,1.5 \mathrm{~Hz}$, $1 \mathrm{H}), 8.00(\mathrm{~d}, J=1.1 \mathrm{~Hz}, 1 \mathrm{H}), 7.65(\mathrm{dd}, J=8.4,1.5 \mathrm{~Hz}$, $1 \mathrm{H}), 7.49(\mathrm{~d}, J=8.5 \mathrm{~Hz}, 1 \mathrm{H}), 7.42-7.18(\mathrm{~m}, 6 \mathrm{H}), 6.68$ $(\mathrm{d}, J=15.7 \mathrm{~Hz}, 1 \mathrm{H}), 6.50-6.34(\mathrm{~m}, 1 \mathrm{H}), 4.63(\mathrm{t}, J=$ $7.0 \mathrm{~Hz}, 1 \mathrm{H}), 4.25(\mathrm{~d}, J=7.4 \mathrm{~Hz}, 2 \mathrm{H}), 4.13(\mathrm{t}, J=7.3$ $\mathrm{Hz}, 2 \mathrm{H}), 3.96$ (s, 3H), 3.23 (t, $J=5.6 \mathrm{~Hz}, 2 \mathrm{H}), 3.18$ (s, $3 \mathrm{H}), 2.04-1.94(\mathrm{~m}, 3 \mathrm{H}), 1.74-1.62(\mathrm{~m}, 3 \mathrm{H}), 1.00(\mathrm{~d}, J$ $=6.2 \mathrm{~Hz}, 6 \mathrm{H}) \cdot{ }^{13} \mathrm{C} \mathrm{NMR}\left(75 \mathrm{MHz}, \mathrm{CDCl}_{3}\right): \delta 167.7$, $156.1,153.0,143.4,142.6,139.3,137.2,136.4,134.0$, $128.5,127.8,126.4,124.3,124.1,123.7,123.6,123.5$, 
122.0, 118.8, 109.8, 109.2, 68.7, 58.5, 52.0, 42.8, 41.8, 38.1, 35.2, 30.0, 29.6, 25.8, 22.4. IR (KBr): 2953, 1713, 1433, $1301 \mathrm{~cm}^{-1}$. ESI-MS $\mathrm{m} / z: 583(\mathrm{M}+1)$. HRMS $\mathrm{m} / \mathrm{z}$ calcd for $\mathrm{C}_{34} \mathrm{H}_{38} \mathrm{~N}_{4} \mathrm{O}_{3} \mathrm{~S}$ : 582.2665. Found: 582.2690.

2'-(4-Fluoro-benzylsulfanyl)-1-(3-methoxy-propyl)-1'(3-methyl-butyl)-1H, $1^{\prime} H$-[2,5']bibenzoimidazolyl-5-carboxylic Acid Methyl Ester (10s). ${ }^{1} \mathrm{H}$ NMR $(300 \mathrm{MHz}$, $\left.\mathrm{CDCl}_{3}\right): \delta 8.53(\mathrm{~d}, J=1.3 \mathrm{~Hz}, 1 \mathrm{H}), 8.05(\mathrm{dd}, J=8.5,1.5$ $\mathrm{Hz}, 1 \mathrm{H}), 8.02(\mathrm{~d}, J=1.2 \mathrm{~Hz}, 1 \mathrm{H}), 7.65(\mathrm{dd}, J=8.3,1.5$ $\mathrm{Hz}, 1 \mathrm{H}), 7.50(\mathrm{~d}, J=8.5 \mathrm{~Hz}, 1 \mathrm{H}), 7.35-7.44(\mathrm{~m}, 3 \mathrm{H})$, $7.05-6.95(\mathrm{~m}, 2 \mathrm{H}), 4.62(\mathrm{~s}, 2 \mathrm{H}), 4.47(\mathrm{t}, J=6.7 \mathrm{~Hz}, 2 \mathrm{H})$, $4.08(\mathrm{t}, J=7.1 \mathrm{~Hz}, 2 \mathrm{H}), 3.96(\mathrm{~s}, 3 \mathrm{H}), 3.24(\mathrm{t}, J=5.6 \mathrm{~Hz}$, 2H) $3.19(\mathrm{~s}, 3 \mathrm{H}), 2.07-1.95(\mathrm{~m}, 2 \mathrm{H}), 1.80-1.51(\mathrm{~m}, 3 \mathrm{H})$, $0.98(\mathrm{~d}, J=6.0 \mathrm{~Hz}, 6 \mathrm{H}) \cdot{ }^{13} \mathrm{C}$ NMR $\left(75 \mathrm{MHz}, \mathrm{CDCl}_{3}\right): \delta$ $167.5,163.7,160.4,155.9,152.7,144.2,143.2,139.0,136.9$, $132.5,130.7,130.6,124.2,123.9,123.4,123.3,121.8,118.8$, 115.5, 115.2, 109.7, 109.1, 68.6, 58.4, 51.9, 42.6, 41.7, 37.8, 36.0, 29.8, 25.6, 22.2. IR (KBr): 2955, 1714, 1433, 1301 $\mathrm{cm}^{-1}$. ESI-MS $\mathrm{m} / \mathrm{z}: 575(\mathrm{M}+1)$. HRMS $\mathrm{m} / \mathrm{z}$ calcd for $\mathrm{C}_{32} \mathrm{H}_{35} \mathrm{~N}_{4} \mathrm{O}_{3} \mathrm{~S}$ : 574.2414. Found: 574.2366.

1-(3-Methyl-butyl)-2' -(naphthalen-2-ylmethylsulfanyl)$1^{\prime}$-thiophen-2-ylmethyl-1H,1'H-[2,5']bibenzoimidazolyl-5carboxylic Acid Methyl Ester (10t). ${ }^{1} \mathrm{H}$ NMR $(300 \mathrm{MHz}$, $\left.\mathrm{CDCl}_{3}\right): \delta 8.55(\mathrm{~d}, J=1.2 \mathrm{~Hz}, 1 \mathrm{H}), 8.12-8.00(\mathrm{~m}, 2 \mathrm{H})$, $7.88(\mathrm{~s}, 1 \mathrm{H}), 7.84-7.76(\mathrm{~m}, 3 \mathrm{H}), 7.65(\mathrm{dd}, J=8.3,1.5 \mathrm{~Hz}$, $1 \mathrm{H}), 7.56(\mathrm{dd}, J=8.5,1.7 \mathrm{~Hz}, 1 \mathrm{H}), 7.53-7.43(\mathrm{~m}, 4 \mathrm{H})$, $7.21(\mathrm{dd}, J=5.0,1.2 \mathrm{~Hz}, 1 \mathrm{H}), 6.96(\mathrm{~d}, J=3.0 \mathrm{~Hz}, 1 \mathrm{H})$, $6.89(\mathrm{dd}, J=8.5,1.5 \mathrm{~Hz}, 1 \mathrm{H}), 5.43(\mathrm{~s}, 2 \mathrm{H}), 4.85(\mathrm{~s}, 2 \mathrm{H})$, 4.33 (t, $J=7.8 \mathrm{~Hz}, 2 \mathrm{H}), 3.98$ (s, 3H), 1.74 (q, $J=7.0 \mathrm{~Hz}$, $2 \mathrm{H}), 1.65-1.50(\mathrm{~m}, 1 \mathrm{H}), 0.88(\mathrm{~d}, J=6.5 \mathrm{~Hz}, 6 \mathrm{H}) .{ }^{13} \mathrm{C} \mathrm{NMR}$ $\left(75 \mathrm{MHz}, \mathrm{CDCl}_{3}\right): \delta 168.1,156.4,153.4,143.9,143.2,139.3$, 137.9, 137.2, 134.3, 133.7, 133.2, 129.0, 128.4, 128.2, 128.1, $127.4,127.3,127.2,126.7,126.6,126.4,124.8,124.5,122.6$, 119.6, 110.1, 52.5, 43.9, 43.2, 38.9, 38.3, 26.4, 22.7. IR ( $\mathrm{cm}^{-1}$, neat): $2953,1711,1433,1301$. ESI-MS $\mathrm{m} / \mathrm{z}: 631$ (M $+1)$. HRMS $m / z$ calcd for $\mathrm{C}_{37} \mathrm{H}_{34} \mathrm{~N}_{4} \mathrm{O}_{2} \mathrm{~S}_{2}: 630.2123$. Found: 630.2133 .

Acknowledgment. The authors thank the National Science Council of Taiwan for financial support and National Chiao Tung University for providing laboratory facilities. We are also grateful to the National Center for High-Performance Computing for computer time and facilities.

Supporting Information Available. Proton and carbon NMR spectra of compounds $6 \mathbf{a}-\mathbf{f}, \mathbf{8} \mathbf{a}-\mathbf{i}$, and $\mathbf{1 0 a}-\mathbf{i}$. This information is available free of charge via the Internet at http://pubs.acs.org.

\section{References and Notes}

(1) Langer, P.; Doring, M. Eur. J. Org. Chem. 2002, 221-234.

(2) (a) Dolle, R. E. J. Comb. Chem . 2003, 5, 693-753. (b) Dolle, R. E. J. Comb. Chem. 2004, 6, 623-679. (c) Dolle, R. E. J. Comb. Chem. 2005, 7, 739-798. (d) Dolle, R. E.; Le Bourdonnec, B.; Morales, G. A.; Moriarty, K. J.; Salvino, J. M. J. Comb. Chem. 2006, 8, 597-635.

(3) (a) Horton, D. A.; Bourne, G. T.; Smythe, M. L. Chem. Rev. 2003, 103, 893-930. (b) Solid Phase Synthesis and Combinatorial Chemistry; Seneci, P., Ed.; Wiley Interscience: New York, 2000. (c) Organic Synthesis on Solid Phase; Dörwald, F. Z., Ed.; Wiley-VCH: Weinheim, Germany, 2000.
(4) (a) Gravert, D. J.; Janda, K. D. Curr. Opinion. Chem. Biol. 2002, 6, 339-346. (b) Swamy, K. M. K.; Yeh, W. B.; Lin, M. J.; Sun, C. M. Curr. Med. Chem. 2003, 10, 2403-2424. (c) Sun, C. M. Soluble Polymer-Supported Synthesis of Heterocyclic Libraries. In Combinatorial Chemistry Methods and Protocols; Bellavance, L., Ed.; Methods in Molecular Biology Series; The Humana Press Inc.: Clifton, NJ, 2002; Chapter 10, pp 345-371. (d) Sun, C. M. Diversity Oriented Synthesis of Biologically Privileged Scaffolds by Microwave Combinatorial Approach. In Frontiers of Medicinal Chemistry; Reitz, A. B., Ed.; Bentham Science Publishers, Ltd.: Bussum, The Netherlands, 2005; Vol. 1, pp 485-510.

(5) Sun, C. M. Lett. Drug Des. Discoverv 2005, 2, 184-193.

(6) Chen, C. H.; Chang, C. M.; Chen, H. Y.; Lai, J. J.; Sun, C. M. J. Comb. Chem. 2007, 9, 618-626.

(7) Chang, W. J.; Yeh, W. B.; Sun, C. M. Synlett 2003, 16881692.

(8) (a) Yeh, W. B.; Sun, C. M. J. Comb. Chem. 2004, 6, 279282. (b) Yeh, W. B.; Lin, M. J.; Lee, M. J.; Sun, C. M. Mol. Diversity 2003, 7, 185-198.

(9) Tung, C. L.; Sun, C. M. Tetrahedron Lett. 2004, 45, 11591162.

(10) Yeh, W. B.; Lin, M. J.; Sun, C. M. Tetrahedron Lett. 2003, 44, 4923-4926.

(11) (a) Velik, J.; Baliharova, V.; Fink-Gremmels, J.; Bull, S.; Lamka, J.; Skalova, L. Res. Vet. Sci. 2004, 76, 95-103. (b) Shin, J. M.; Cho, Y. M.; Sachs, J. J. Am. Chem. Soc. 2004, 126, 7800-7811. (c) Mason, J. S.; Morize, I.; Menard, P. R.; Cheney, D. L.; Hulme, C.; Labaudiniere, R. F. J.Med. Chem. 1999, 42, 3251-3264. (d) Spasov, A. A.; Yozitsa, I. N.; Bugaeva, L. I.; Anisimova, V. A. Pharm. Chem. J. 1999, 33, 232-244. (e) Gravatt, G. L.; Baguley, B. C.; Wilson, W. R.; Denny, W. A. J. Med. Chem. 1994, 37, 4338-4345. (f) Porcari, A. R.; Devivar, R. V.; Kucera, L. S.; Drach, J. C.; Townsend, L. B. J.Med. Chem. 1998, 41, 1252-1262. (g) Backlund, M.; Weidolf, L.; Sundberg, M. I. Eur. J. Biochem. 1999, 261, 6671. (h) Starčević, K.; Kralj, M.; Ester, K.; Sabol, I.; Grce, M.; Pavelić, K.; Karminski-Zamola, G. Bioorg. Med. Chem. 2007, 15, 4419-4426. (i) Salluja, S.; Zou, R.; Drach, J. C.; Townsend, L. B. J. Med. Chem. 1996, 39, 881-891.

(12) (a) Ramla, M. M.; Omar, M. A.; Tokuda, H.; El-Diwani, I. Bioorg. Med. Chem. 2007, 15, 6489-6496. (b) NavarreteVázquez, G.; Rojano-Vilchis, M. M.; Yépez-Mulia, L.; Meléndez, V.; Gerena, L.; Hernández-Campos, A.; Castillo, R.; Hernández-Luis, F. Eur. J. Med. Chem. 2006, 41, 135141. (c) Rivara, M.; Zuliani, V.; Cocconcelli, G.; Morini, M.; Comini, M.; Rivara, S.; Mor, M.; Bordi, F.; Barocelli, E.; Ballabeni, V.; Bertoni, S.; Plazzi, P. V. Bioorg. Med. Chem. 2006, 14, 1413-1424. (d) Safonov, I. G.; Heerding, D. A.; Keenan, R. M.; Price, A. T.; Erickson-Miller, C. L.; Hopson, C. B.; Levin, J. L.; Lord, K. A.; Tapley, P. M. Bioorg. Med. Chem. Lett. 2006, 16, 1212-1216.

(13) (a) Wu, Z; Rea, P.; Wickham, G. Tetrahedron Lett. 2000, 41, 9871-9874. (b) Vourloumis, D.; Takahashi, M.; Simonsen, K. B.; Ayida, B. K.; Barluenga, S.; Winters, G. C.; Hermann, T. Tetrahedron Lett. 2003, 44, 2807-2811. (c) Roy, A. D.; Sharma, S.; Grover, R. K.; Kundu, B.; Roy, R. Org. Lett. 2004, 6, 4763-4766. (d) Hioki, H.; Matsuhita, K.; Kubo, M.; Harada, K.; Kodama, M.; Fukuyama, Y. Tetrahedron 2007, 63, 1131511324.

(14) (a) Yeh, C. M.; Tung, C. L.; Sun, C. M. J. Comb. Chem. 2000, 2, 341-348. (b) Bendale, P. M.; Sun, C. M. J. Comb. Chem. 2002, 4, 359-361. (c) Lin, M. J.; Sun, C. M. Sunlett 2004, 663-666. (d) Chen, C.; Chen, Y. J. Tetrahedron Lett. 2004, 45, 113-115. (e) Yeh, W. B.; Lin, M. J.; Sun, C. M. Comb. Chem. High Throughput Screening 2004, 7, 251-255. (f) Su, Y. S.; Lin, M. J.; Sun, C. M. Tetrahedron Lett. 2005, $46,177-180$.

(15) (a) Alexandra, J.; Sun, X. W.; Eric, J.; Christian, B.; John, M.; Stephen, N. Biochemistry 2003, 42, 5984-5992. (b) Ji, Y. H.; Bur, D.; Hasler, W.; Schimitt, V. R.; Dorn, A.; Bailly, 
C.; Waring, M. J.; Hochstrasser, R.; Leupin, W. L. Bioorg. Med. Chem. 2001, 9, 2905-2919. (c) Tanious, D.; Hamelberg, D.; Bailly, A.; Czamy, D.; Boykin, W. D.; Wilson, W. D. J. Am. Chem. Soc. 2004, 126, 143-153.

(16) (a) Kakkar, R.; Garg R., Suruchi. J. Mol. Struct. Theochem. 2002, 584, 37-44. (b) Chaudhari, P.; Ganguly, B.; Bhattacharya, S. J. Org. Chem. 2007, 72, 1912-1923.

(17) (a) Jin, S.; Kim, J. S.; Sim, S. P.; Liu, A.; Pilch, D. S.; Liu, L. F.; LaVoie, E. J. Bioorg. Med. Chem. Lett. 2000, 10, 719 723. (b) Alper, S.; Arpaci, O. T.; Aki-Sener, E. S.; Yalcin, Y. Il Farmaco 2003, 58, 497-507.

(18) (a) Seaton, A.; Higgins, C.; Mann, J.; Baron, A.; Bailly, C.; Neidle, S.; Van den Berg, H. Eur. J. Cancer 2003, 39, 25482555. (b) Rastogi, K.; Chang, J. Y.; Pan, W. Y.; Chou, C. H.; Chen, T. C.; Su, T. L. J. Med. Chem. 2002, 45, 4485-4493.

(19) Brandstrom, A.; Lindberg, P.; Jungren, U. Scand. J. Gastroenterol. Suppl. 1986, 21, 54-59.

(20) Doerge, D. R.; Decker, C. J.; Takazawa, R. S. Biochemistrv 1993, 32, 58-65.

(21) Decker, C. J.; Doerge, D. R.; Cashmann, J. R. Chem. Res. Toxicol. 1992, 5, 726-733.

(22) Nishigori, H.; Sato, M.; Nishimura, C. Arch. Virol. 1978, 58, 335-340.
(23) Kosano, H.; Kayanuma, T.; Nishigori, H. Biochem. Biophys. Acta. Mol. Cell. Res. 2000, 1499, 11-18.

(24) Corban, G. J.; Hadjikakou, S. K.; Hadjiliadis, N.; Kubicki, M.; Tiekink, E. T. R.; Butler, I. S.; Drougas, E.; Kosmas, A. M. Inorg. Chem. 2005, 44, 8617-8627.

(25) Yeh, C. M.; Sun, C. M. Tetrahedron Lett. 1999, 40, 72477250 .

(26) Block, F. A.; Logan, M. S. P.; Baoli, C.; Wackett, L. P. J. Biol. Chem. 1994, 269, 8826-8830.

(27) Pan, P. C.; Sun, C. M. Tetrahedron Lett. 1999, 40, 64436446.

(28) Anklekar, K. Y.; Kulkarni, M. V. Indian J. Chem. 1995, 34B, 677-378.

(29) Varma, R. S. J. Ind. Chem. Soc. 2004, 81, 627-639.

(30) (a) Hwu, J. R.; Singha, R.; Hong, S. C.; Chang, Y. H.; Das, A. R.; Vliegen, I.; DeClercq, E.; Neyts, J. Antiviral Res. 2007, 77, 157-162. (b) Andrzejewska, M.; Yepez-Mulia, L.; Tapia, A.; Cedillo-Rivera, R.; Laudy, A. E.; Staršciak, B. J.; Kazimierczuk, Z. Eur. J. Pharm. Sci. 2004, 21, 323329.

(31) Dou, H. J. M.; Ludwikow, M.; Hassanaly, P.; Kister, J.; Metzer, J. J.Heterocycl. Chem. 1980, 17, 393-395.

CC7002045 